\title{
Perfil epidemiológico da tuberculose em Juiz de Fora, município prioritário no estado de Minas Gerais
}

\section{Hיtrista}

Epidemiological profile of tuberculosis in Juiz de Fora, priority

municipality in Minas Gerais state

${ }^{1}$ Universidade Federal de Juiz de Fora, Hospital Universitário, Laboratório de Análises Clínicas Juiz de Fora, MG.

\section{Renata Rêgo}

Rua Dr. Pedro de Aquino Ramos,

50/407.

Granbery, Juiz de Fora-MG

CEP: $36010-440$

७ renata_torresrtr@hotmail.com

\section{RESUMO}

Introdução: A tuberculose (TB) é considerada um dos maiores problemas mundiais de saúde. Visando a eliminação dessa doença, a Organização Mundial de Saúde (OMS) elaborou um plano, chamado "The end TB strategy", cujas metas propostas foram adotadas pelo Ministério da Saúde. Juiz de Fora é a segunda cidade do estado de Minas Gerais com os maiores números de casos dessa doença sendo considerada município prioritário para controle da TB. Objetivo: Realizar uma análise do perfil epidemiológico da TB na cidade de Juiz de Fora nos anos de 2008 a 2017, comparando os dados registrados a nível nacional, estadual e municipal, bem como avaliar o cenário municipal frente às metas propostas pelo plano de enfrentamento da TB. Material e métodos: Foi realizado um estudo transversal, descritivo, de série temporal utilizando dados secundários disponibilizados pelo Departamento de Informática do Sistema Único de Saúde (DATASUS). Resultados: O coeficiente de incidência e mortalidade da TB assim como a taxa de abandono de tratamento verificados em Juiz de Fora, de 2008-2017, superaram as médias nacionais e estaduais, além de extrapolarem as metas previstas no plano de enfrentamento da TB. Cabe ressaltar, no entanto, que a proporção de teste rápido de HIV e o número de cultura de escarro realizados entre os casos de retratamento de TB superaram as médias nacional e estadual. Conclusão: O perfil epidemiológico da TB em Juiz de Fora revelou uma situação em alguns aspectos piores em relação ao cenário nacional e estadual, mas também evidenciou que medidas já estão sendo realizadas pelo município para o enfrentamento da doença.

Palavras-chave: tuberculose, epidemiologia, saúde pública

\section{ABSTRACT}

Introduction: Tuberculosis (TB) is considered one of the biggest global health problems. In order to eliminate this disease, the World Health Organization (WHO) elaborated a plan, called The end TB strategy, whose goals were implemented by the Brazilian National Ministry of Health. Juiz de Fora is the second city in Minas Gerais with higher numbers of disease cases that is being considered a priority municipality for the TB control. Objectives: Analyze the epidemiological profile of TB in Juiz de Fora from 2008 to 2017, comparing data recorded at national, state and municipal level, as well as to access the municipal scenario against the targets proposed by the WHO plan. Material and methods: A crosssectional, descriptive, time series study was performed using secondary data provided by the Brazilian Unified Health System's Department of Informatics (DATASUS). Results: The TB incidence and mortality coefficient as well as treatment drop-out rate observed in Juiz de Fora, from 2008-2017, exceeded national and state averages, in addition to extrapolating the targets set in the WHO plan. It should be noted, however, that the proportion of rapid HIV test and the number of sputum culture performed among retreatment TB cases exceeded national and state averages. Conclusion: In some aspects, the TB epidemiological profile in Juiz de Fora revealed a situation worse than national and state scenario, but also showed that arrangements are already being carried out by the municipality to confront the disease.

Keywords: tuberculosis, epidemiology, public health 


\section{INTRODUÇÃO}

A tuberculose (TB) é uma doença infecciosa causada pelo bacilo Mycobacterium tuberculosis. Apesar de acometer a humanidade há milênios, essa enfermidade permanece como um dos maiores problemas mundiais de saúde. Em 2016 foram notificados aproximadamente 10 milhões de casos novos de TB, que ocupa o nono lugar, mundialmente, entre as doenças infecciosas de patógeno único que mais matam, ultrapassando o número de mortes causadas pelo vírus da imunodeficiência humana (HIV) (OMS, 2017).

De acordo com a Organização Mundial de Saúde (OMS, 2017), o Brasil se encontra na lista dos países com os maiores números de incidência de TB, além de estar também entre os 20 países com a maior incidência de TB entre as pessoas vivendo com HIV. O último boletim epidemiológico do Ministério da Saúde (MS) (2018) mostrou que os estados com maiores coeficientes de incidência foram Amazonas (74,1/100 mil hab.), Rio de Janeiro (63,5/100 mil hab.) e Pernambuco (46,0/100 mil hab.).

Entre os estados da região Sudeste, deve-se destacar que Minas Gerais apresentou em 2017 o menor coeficiente de incidência de TB (15,8/100 mil hab.). Entretanto, possui 11 municípios prioritários para o Programa Nacional de Controle da Tuberculose (PNCT). Esse programa integrado à rede de Serviços de Saúde preconiza a distribuição gratuita de medicamentos e ações preventivas e de controle da TB como, por exemplo, a implantação do tratamento diretamente observado (TDO) (BRASIL, 2014; BRASIL, 2018a).

Juiz de Fora, localizada na Zona da Mata mineira, é a segunda cidade do estado de Minas Gerais com os maiores números de casos de TB notificados no Sistema de Informação de Agravos de Notificação (SINAN). Em 2017 apresentou uma incidência de 42 casos por 100 mil habitantes, valor aproximadamente 2,7 vezes maior do que a incidência no Estado. Por essa razão, é considerado município prioritário em relação a TB (BRASIL, 2014; BRASIL, 2018; DATA-SUS 2018).

A OMS visando a eliminação da TB no mundo, elaborou uma estratégia global para o enfrentamento dessa doença, intitulada "The end TB strategy", anunciada na Assembleia Mundial de Saúde em 2014. Seguindo as diretrizes da OMS, o MS por meio do PNCT, criou o Plano Nacional pelo Fim da Tuberculose como Problema de Saúde Pública com a meta de reduzir o coeficiente de incidência no Brasil em menos de 10 casos por 100 mil habitantes e o coeficiente de mortalidade para menos de 1 óbito por 100 mil habitantes até o ano de 2035 (BRASIL, 2017).

Para o monitoramento da execução do plano, o PNCT elaborou uma lista de indicadores prioritários para serem utilizados pelos estados e municípios como forma de verificação do desenvolvimento das ações (BRASIL, 2017). Foram escolhidos cinco desses indicadores para a execução desse trabalho sendo eles: coeficiente de incidência de TB todas as formas; coeficiente de mortalidade por TB; proporção de testagem para HIV entre os casos novos de TB; proporção de abandono de tratamento de casos novos de TB pulmonar com confirmação laboratorial e proporção de realização de cultura de escarro entre os casos de retratamento da TB.

Considerando o impacto da TB no cenário mundial e tendo em vista as metas da OMS, o objetivo do estudo foi realizar uma análise do perfil da TB na cidade de Juiz de Fora - MG, no período de 2008 a 2017, comparando os resultados municipais com os indicadores nacionais e estaduais bem como avaliar o cenário municipal com as metas propostas pelo plano nacional de enfrentamento da TB.

\section{MATERIAL E MÉTODOS}

O presente trabalho trata-se de um estudo transversal, descritivo, de série temporal. Utilizou-se dados secundários obtidos através dos bancos de dados SINAN (Sistema de Informações de Agravos e Notificação) e SIM (Sistema de Informação sobre Mortalidade) disponibilizados pelo Departamento de Informática do Sistema Único de Saúde (DATASUS). Além disso as estimativas populacionais, do período de 2008 a 2015, utilizadas no cálculo dos indicadores epidemiológicos foram extraídas da Rede Integrada de Informações em Saúde (RIPSA IBGE) também disponibilizada no DATASUS e as estimativas do período de 2016 a 2018 do site do Instituto Brasileiro de Geografia e Estatística (IBGE). Por se tratar de bancos de dados de domínio público, as informações de identificação não foram disponibilizadas, o que assegura o sigilo e anonimato das pessoas com tuberculose, não sendo, portanto, necessário submeter o projeto ao Comitê de Ética em Pesquisa.

A população de estudo foi constituída por todos os casos novos de TB registrados no período de 2008 a 2017 no Brasil (716.485 casos novos), em Minas Gerais (37.371 casos novos) e em Juiz de Fora (1.987 casos novos). No DATASUS, o caso de TB é categorizado em 6 tipos de entrada: caso novo, recidiva, reingresso após abandono, não sabe, transferência e pós óbito. De acordo com o Ministério da Saúde (2016) a categoria caso novo é definido como "qualquer caso que nunca utilizou a medicação antituberculosa, ou a utilizou por menos de 30 dias". Por isso, para o cálculo dos indicadores é considerado como critério de inclusão todos os casos classificados como caso novo, não sabe (caso com história prévia desconhecida) e pós-óbito (caso descoberto ou notificado após a morte do paciente). Excluiu-se todos os casos com registro de mudança de diagnóstico na situação de encerramento. Os dados de 2001 a 2013 foram atualizados em outubro de 2017 e dos anos 2014 a 2017 atualizados em maio de 2018, estando, ainda, sujeitos à revisão.

O coeficiente de incidência foi calculado pelo número de casos novos por 100 mil habitantes na população residente e o coeficiente de mortalidade pelo número de óbitos por 100 mil habitantes. As informações disponíveis sobre 
estatísticas vitais no DATASUS abrangem mortalidade no período de 1996 a 2016 e dados preliminares de 2017. Os números de óbitos foram obtidos considerando todas as formas da doença listadas na CID-10 com os códigos A-15 a A-19 (CONASS, 2018) e selecionando o conteúdo "óbitos por residência".

Foram considerados como "realizados" os testes para HIV com resultado positivo e negativo, sendo desconsiderados os que estavam em andamento e ignorado ou em branco. O percentual de abandono foi estudado em relação aos casos novos de TB da forma pulmonar com situação de encerramento declarada como abandono. Por fim, foi avaliada a porcentagem de cultura de escarro realizada (com resultado positivo, negativo e em andamento) entre os casos de retratamento (categorizados no DATASUS como recidiva e reingresso após abandono), excluindo-se os resultados ignorados ou em branco.

Todos os cálculos e gráficos foram gerados utilizando o programa estatístico Microsoft ${ }^{\circledR}$ Office Excel.

\section{RESULTADOS}

O coeficiente médio anual de incidência da TB em Juiz de Fora para o período estudado foi de aproximadamente 36,5 casos/100.000 habitantes (Figura 1 ). O resultado observado aproximou-se da média nacional (35,9 casos/100.000 habitantes), mas destoou da média estadual (18,3 casos/ 100.000 habitantes). Quando se analisa a evolução do coeficiente de incidência comparando o ano de 2008 e 2017 percebe-se que Juiz de Fora apresentou um aumento de $17,6 \%$ diferindo da tendência de queda observada no Brasil e em Minas Gerais de 9,3\% e 27,6\% respectivamente.

O coeficiente de mortalidade por TB em Juiz de Fora oscilou durante os anos de estudo apresentando um valor médio de 2,4 óbitos/ 100 mil habitantes. De 2008 a 2017 ocorreu uma redução de $10 \%$ no indicador municipal, $26 \%$ a nível estadual e, nacionalmente, o indicador apresentou queda de $12 \%$ (Figura 2).

A análise histórica mostrou um aumento gradual na realização de testagem para o HIV tanto em nível nacional quanto estadual (Figura 3). A análise dos dados municipais apontou uma variação maior durante o período analisado sendo que nos últimos dois anos, a média de realização dos testes foi de aproximadamente $81,0 \%$ superando as médias do Brasil e Minas Gerais.

Com relação ao percentual de abandono de tratamento entre os casos novos de TB pulmonar, o Brasil manteve um indicador médio de 10,7\% até o ano de 2015 e a partir de 2016, sofreu queda, tendo neste ano chegado a 9,8\% e, em 2017, alcançou 9,4\%. A diminuição do abandono também pode ser observada em Minas Gerais que mantinha um indicador de aproximadamente 12,4\% até 2014 sofrendo queda nos anos seguintes. A análise de Juiz de Fora, apesar de apresentar uma maior variação nos dados, com um aumento considerável de 2008 a 2011, mostra que a partir de 2014, o município também apresentou uma queda desse indicador, sendo que de $22,4 \%$ de abandono registrados passou a uma média de $17,6 \%$ nos anos seguintes.

$\mathrm{Na}$ análise histórica de 10 anos descrita na Figura 5, observa-se um aumento da realização do exame de cultura de escarro nos casos de retratamento tanto no Brasil quanto em Minas Gerais. Entretanto, em nenhum período o indicador foi superior a 50\%. Em Juiz de Fora, os dados apresentam grandes oscilações sendo os maiores valores observados em 2016 (64,2\%) e 2017 (70,2\%).

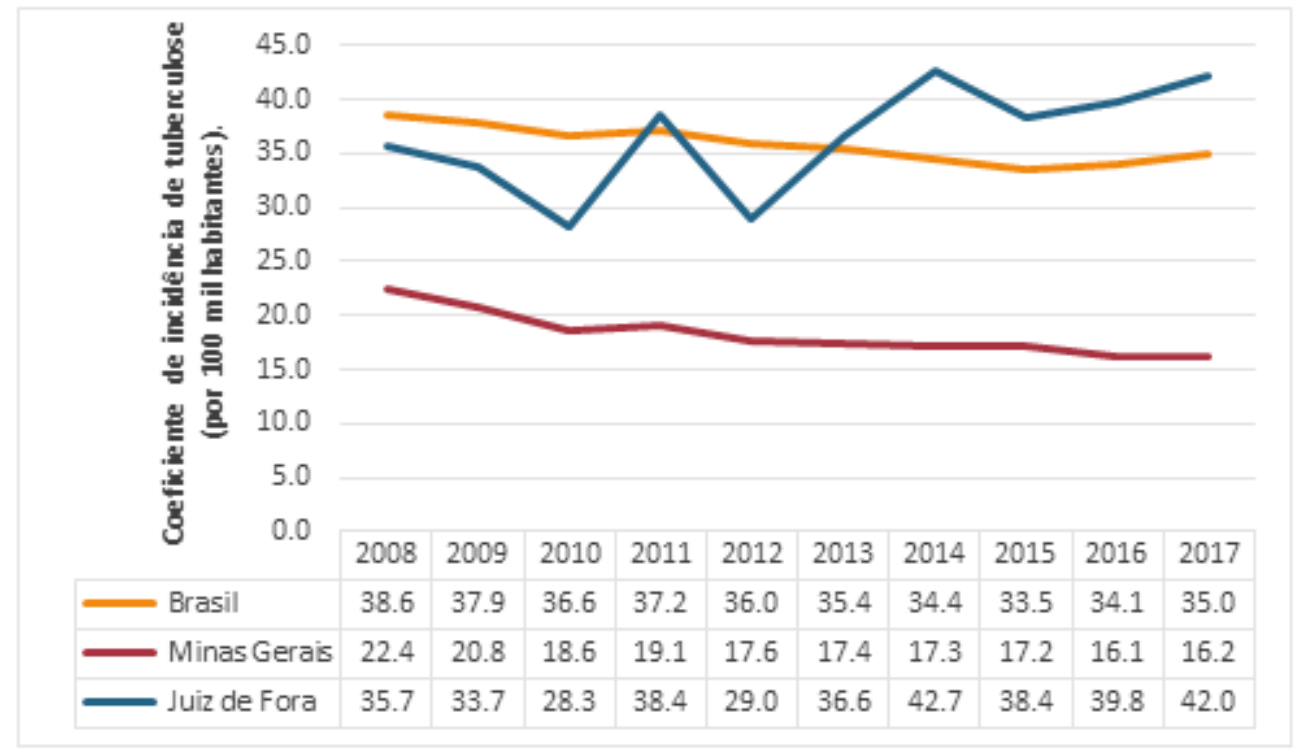

Fonte: Ministério da Saúde/SVS - Sistema de Informação de Agravos de Notificação - Sinan Net (2018)

Figura 1: Coeficiente de incidência de tuberculose (por 100 mil habitantes) no Brasil, Minas Gerais e Juiz de Fora no período de 2008 a 2017 


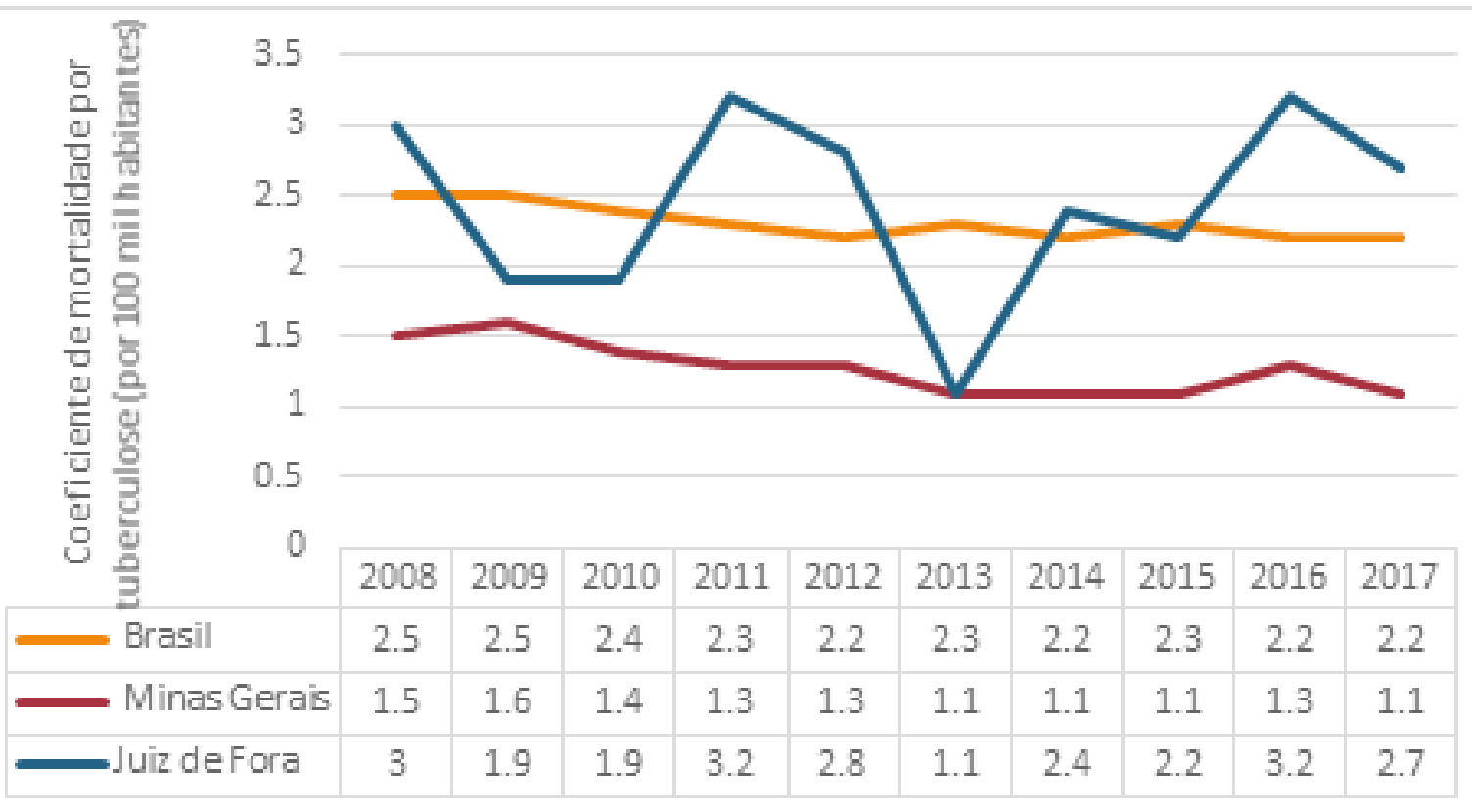

Fonte: MS/SVS/CGIAE - Sistema de Informações sobre Mortalidade - SIM (2018)

Figura 2: Coeficiente de mortalidade de tuberculose (por 100 mil habitantes) no Brasil, Minas Gerais e Juiz de Fora nos anos de 2008 a 2017

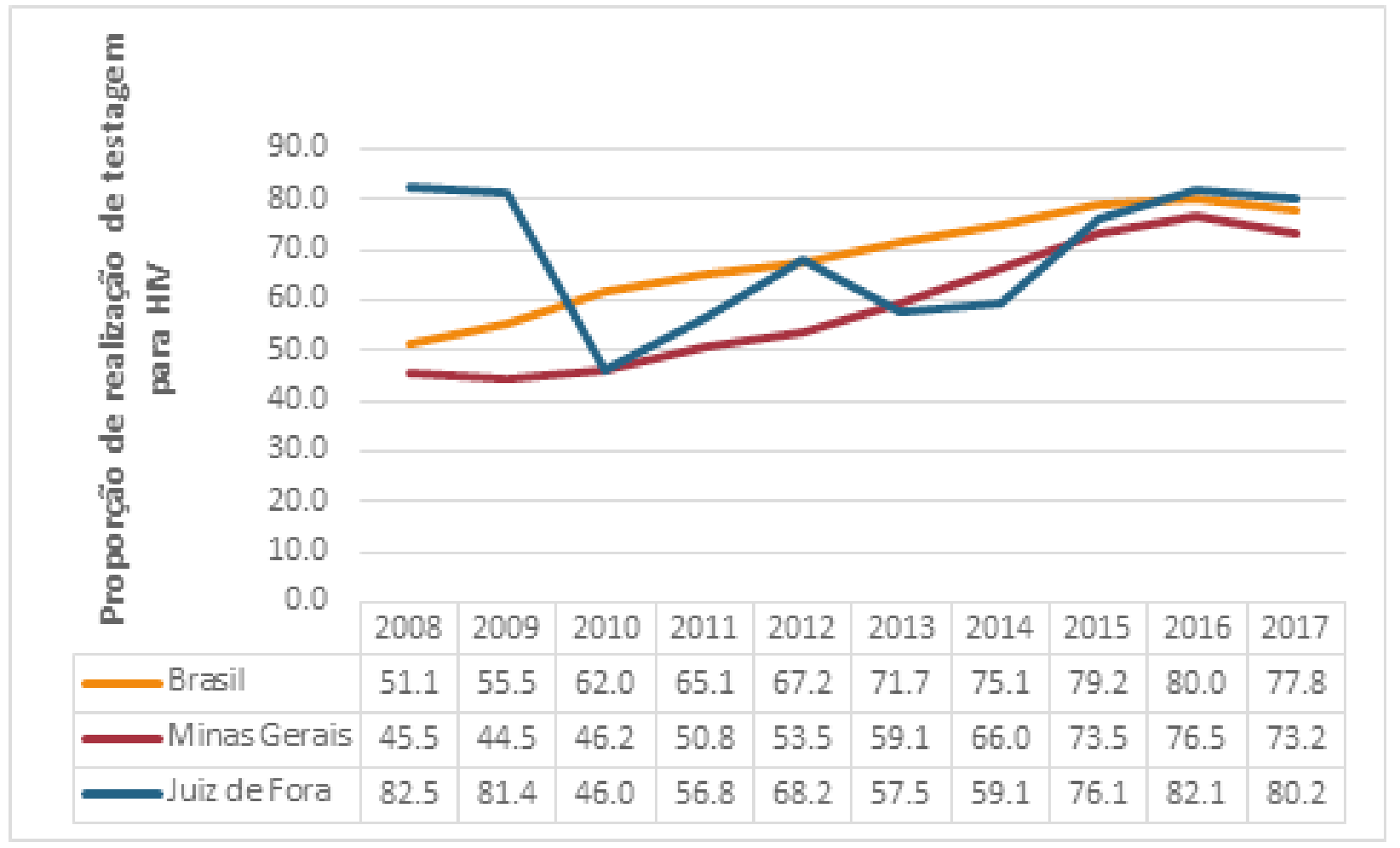

Fonte: Ministério da Saúde/SVS - Sistema de Informação de Agravos de Notificação - Sinan Net (2018)

Figura 3: Porcentagem de testes realizados para HIV entre os casos novos de TB no Brasil, Minas Gerais e Juiz de Fora nos anos de 2008 a 2017 


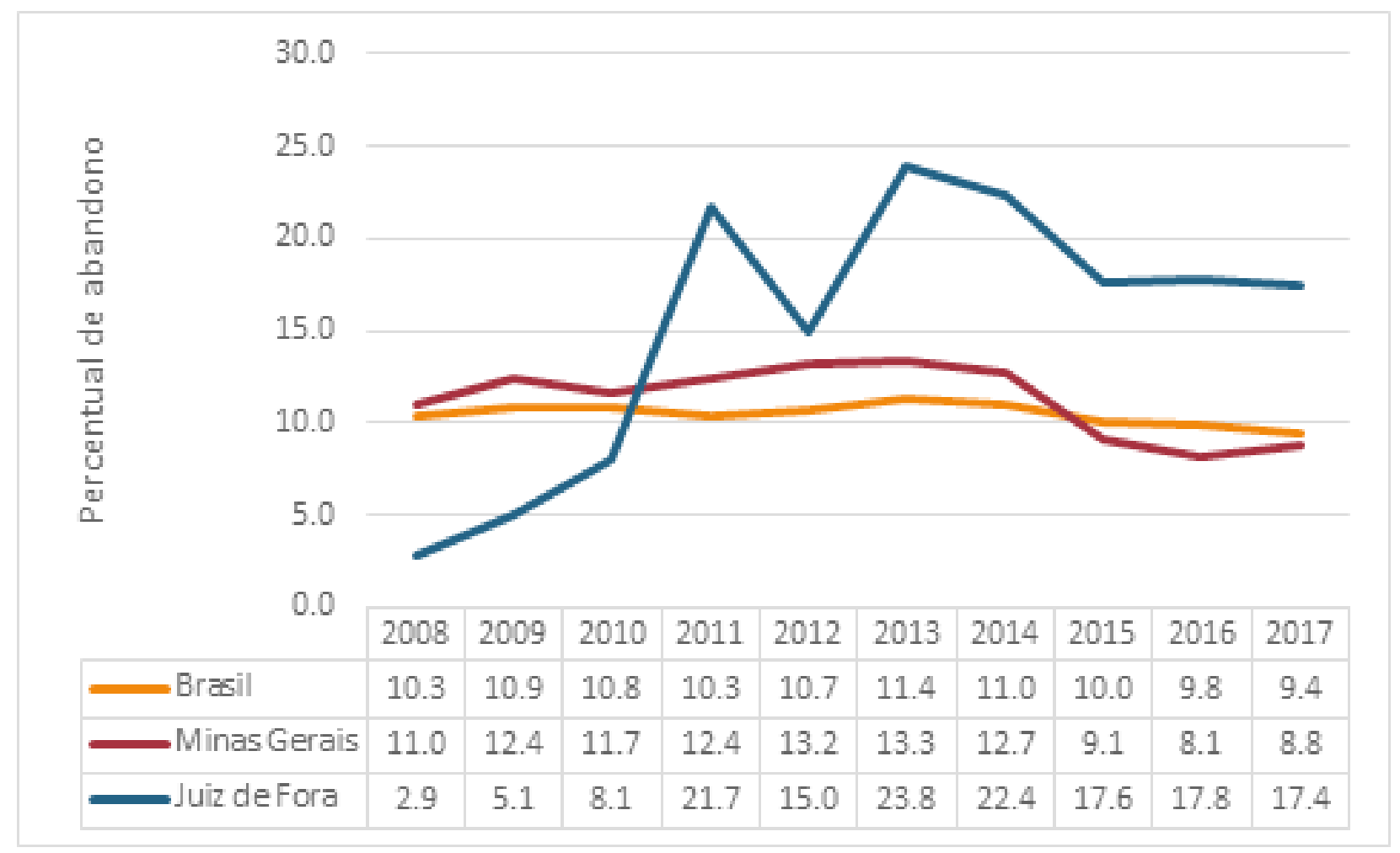

Fonte: Ministério da Saúde/SVS - Sistema de Informação de Agravos de Notificação - Sinan Net (2018)

Figura 4: Porcentagem de abandono de tratamento entre os casos de TB pulmonar no Brasil, Minas Gerais e Juiz de Fora nos anos de 2008 a 2017

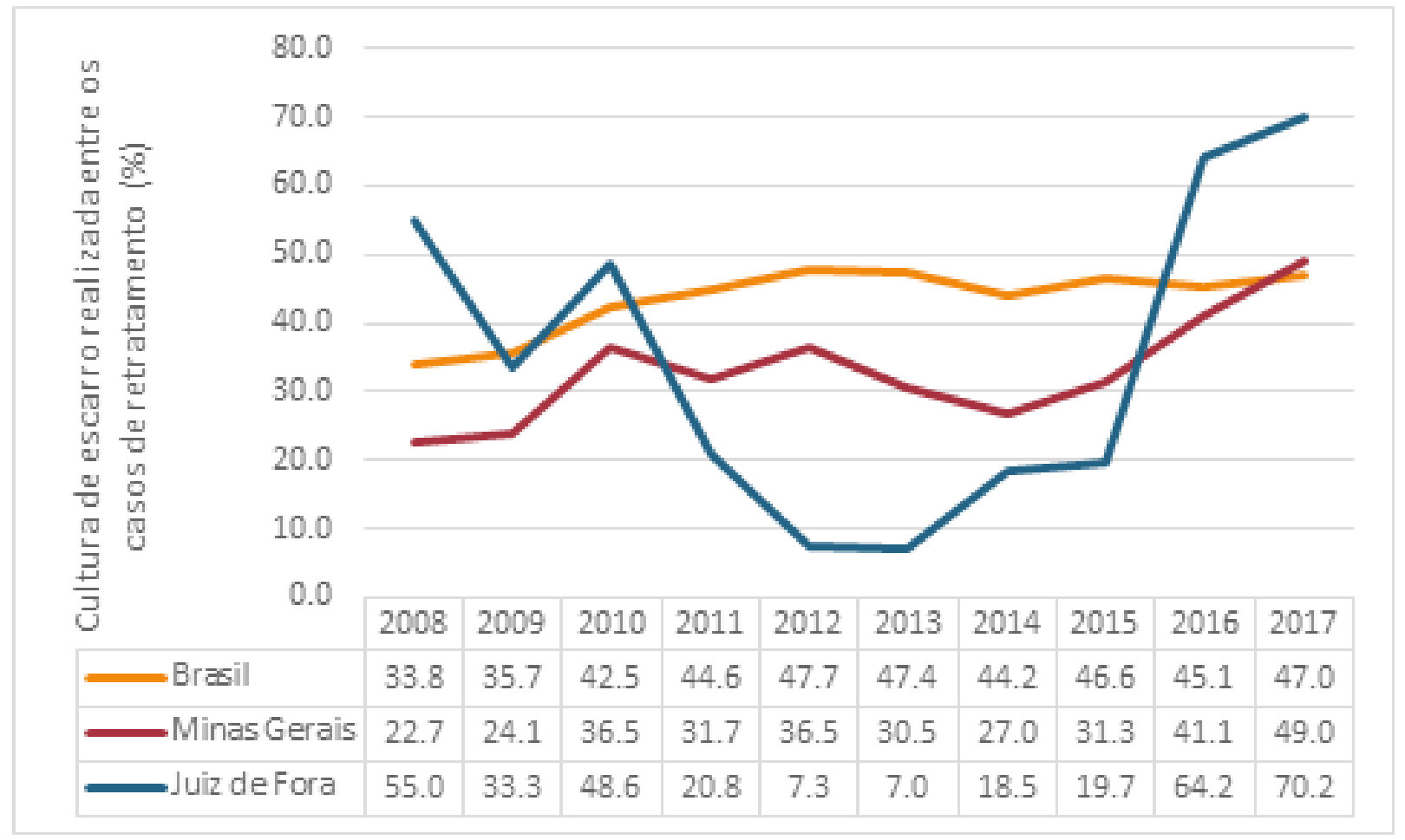

Fonte: Ministério da Saúde/SVS - Sistema de Informação de Agravos de Notificação - Sinan Net (2018).

Figura 5: Porcentagem de cultura de escarro realizada entre os casos de retratamento de TB no Brasil, Minas Gerais e Juiz de Fora nos anos de 2008 a 2017 


\section{DISCUSSÃO}

O Plano Nacional pelo Fim da Tuberculose como Problema de Saúde Pública, implantado pelo Ministério da Saúde, objetiva a eliminação da epidemia dessa doença, tendo como uma das metas a redução do coeficiente de incidência da tuberculose para menos de 10 casos por 100 mil habitantes e do coeficiente de mortalidade para menos de 1 óbito por 100 mil habitantes até o ano de 2035 (BRASIL, 2017). A análise histórica revelou que apesar da redução do coeficiente de incidência observada de 2008 para 2017, Brasil e Minas Gerais ainda não alcançaram a meta descrita no plano. Em Juiz de Fora a situação pode ser considerada pior, pois além do indicador não estar dentro da meta preconizada, verificou-se um aumento da incidência no período estudado. Situação semelhante ocorre com o coeficiente de mortalidade, que apesar da queda registrada, somente em nível estadual o indicador encontra-se próximo a meta, 1,1 óbitos por $100 \mathrm{mil}$ habitantes em 2017. Juiz de Fora registrou uma menor redução do coeficiente de mortalidade comparado aos valores estaduais e nacionais sendo que no último ano analisado foram registrados 2,7 óbitos por $100 \mathrm{mil}$ habitantes, valor superior ao coeficiente nacional $(2,2$ óbitos por 100 mil habitantes).

Lima (2013) destaca que apesar do município adotar estratégias descentralizadoras de controle da TB desde 2007 e da presença de hospitais de referência para o tratamento dessa doença o surgimento de novos casos continua desafiando os profissionais de saúde e os gestores administrativos da secretaria de saúde do município. Esse cenário se justifica pela multicausalidade da gênese e prevalência da doença, relacionados com as condições de pobreza da maioria dos doentes, o baixo conhecimento e atuação dos agentes comunitários de saúde acerca da TB, uma atenção básica precária na cidade, tudo isso somado aos aspectos sociais e epidemiológicos apresentados pelo paciente (LIMA, 2013). Angelo (2008), além de relacionar a pobreza com os altos indicadores de TB em JF destacou também a alta incidência de AIDS na cidade encontrando uma correlação significativa com a incidência da TB nas Regiões Urbanas analisadas em seu trabalho.

De acordo com o Ministério da Saúde (2013) "A tuberculose (TB) ativa em pessoas que vivem com HIV/AIDS (PVHA) é a condição de maior impacto na mortalidade por AIDS e por TB no país". Sendo assim, uma das estratégias do Plano Nacional é oferecer testagem de HIV a todas as pessoas com TB (BRASIL, 2017). Juiz de Fora, assim como Minas Gerais e o Brasil, ainda não atingiu $100 \%$ de testes rápidos realizados, mas é possível observar um aumento na realização desse exame em todas as esferas estudadas. O Plano de Saúde 2014-2017 elaborado pela Secretaria de
Saúde de Juiz de Fora (2013) determinou como meta uma proporção de exames anti-HIV realizados entre os casos novos de tuberculose de $58 \%$, 60\% e $62 \%$ para os anos de 2013, 2014, e 2015 respectivamente. Os resultados desse estudo mostraram que a meta ficou próxima de ser atingida nos anos 2013 (57,5\%) e 2014 $(59,1 \%)$ sendo que em 2015 extrapolou o proposto chegando a $76,1 \%$.

O abandono do tratamento é um grande obstáculo para o controle e eliminação da TB por estar relacionado a uma menor taxa de cura e uma maior chance de um desfecho desfavorável, como o óbito por TB e o aparecimento de micobactérias multirressistentes (PEREIRA, et al., 2018; ROCHA, ADORNO, 2012). A meta para a taxa de abandono pela Organização Mundial de Saúde adotado no Plano Nacional é de 5\%. Pela análise histórica, percebe-se que esse indicador em todos os âmbitos analisados (nacional, estadual e municipal), apesar de apresentar uma queda nos últimos anos, ainda está alto em relação a meta proposta. Frente a essa situação, torna-se necessário uma maior atuação das equipes multiprofissionais em todos os níveis de atenção (primário, secundário e terciário), no desenvolvimento de ações que garantam a adesão do tratamento pelo paciente (MINAS GERAIS, 2019).

Ainda em relação ao abandono do tratamento, observa-se uma grande variação nos dados de JF com um aumento considerável de 2008 a 2011. Isso pode ser justificado pela negligência de informações nos dados notificados ao SINAN pelo município. Pereira et al. (2015), em um estudo de coorte prospectivo com 504 casos notificados no SINAN em Juiz de Fora, comparou as taxas de abandono relatadas no sistema com as taxas de abandono detectadas nos grupos de pacientes acompanhados diretamente pela sua pesquisa. Foi concluído que no período analisado, de 2008 a 2009, a taxa de abandono relatada no SINAN como $3,2 \%$ seriam na realidade $11,9 \%$ indicando uma subnotificação dos dados enviados ao sistema de informação, o que poderia prejudicar ações de combate a esse problema de saúde pública.

Em 2015 foi elaborado e aprovado pela Secretaria Estadual de Saúde, o Plano Municipal de Combate à Tuberculose, em consonância com o Plano Nacional, tendo como um dos objetivos a redução dos casos de TB em Juiz de Fora (ARAÚJO, 2016). Presume-se que os dados notificados no município a partir de então sejam mais fidedignos, visto que foram designados profissionais na atenção primária que seriam responsáveis pelo preenchimento de planilhas e pela verificação da eficácia e qualidade do tratamento dos pacientes (ARAÚJO, 2016).

Por fim, o último indicador analisado foi a proporção de cultura de escarro realizada entre os casos de retratamento. Um dos objetivos do Plano Nacional é oferecer de forma universal a cultura e o teste de 
sensibilidade, além do uso de testes rápidos de forma que o diagnóstico de todas as formas de tuberculose seja realizado mais precocemente (BRASIL, 2017). No Brasil e em Minas Gerais observa-se um aumento no número de culturas realizadas no decorrer dos anos estudados, entretanto, em mais da metade dos casos de retratamento esse exame ainda não está sendo realizado. Em Juiz de Fora, observa-se uma ampliação no número de culturas realizadas a partir de 2015, atingindo aproximadamente $70 \%$ de exames realizados em 2017.

A cultura de escarro ainda é considerada o padrão ouro para o diagnóstico das infecções por micobactérias. Entretanto, a sua execução pelo método padrão de descontaminação da amostra (método Petroff modificado) exige uma estrutura laboratorial de biossegurança nível 3 e profissionais mais qualificados, o que pode ser um fator limitante que explicaria os baixos números de cultura realizados no país (COSTA, et al. 2018; SULIS, et al.; 2016). Visando ampliar a oferta e o acesso a esse exame, o Ministério da Saúde tem realizado capacitação e fornecido suporte técnico-financeiro em algumas capitais e municípios estratégicos, para a implantação do método de cultura Ogawa-Kudoh que dispensa o uso de alguns equipamentos tornando o exame mais simples e econômico (BRASIL, 2011).

Em Minas Gerais, ocorreu uma descentralização do exame de cultura de escarro para os cinco Laboratórios Macrorregionais, um deles localizado em Juiz de Fora, e foi estabelecido critérios e fluxos relacionados ao diagnóstico laboratorial da TB no estado. Essas ações contribuíram para a melhora do indicador nos últimos anos, mas ainda há uma necessidade de ações para o fortalecimento da Rede Laboratorial no Estado uma vez que o indicador ainda segue abaixo do estabelecido pelo Plano Nacional (MINAS GERAIS, 2019).

Em linhas gerais, acredita-se que o Brasil possa liderar o movimento global para eliminar a tuberculose uma vez que possui um sistema de saúde universal gratuito estruturado e com condições técnicas para tal, sendo necessário a priorização por parte do governo dos investimentos em saúde, na articulação intersetorial, no incentivo à participação social e em políticas públicas que combatam a determinação social da doença (BARREIRA, 2018).

\section{CONCLUSÃO}

A TB é uma realidade no município de Juiz de Fora e várias melhorias precisam ser realizadas para que se alcancem as metas propostas no Plano Nacional pelo Fim da Tuberculose como Problema de Saúde Pública. A análise do perfil epidemiológico mostrou que o coeficiente de incidência e mortalidade da doença no município estão acima dos valores do estado e do país.
O estudo da porcentagem de abandono de tratamento revelou uma situação preocupante visto que, Juiz de Fora apresentou um percentual alto de abandono em relação a meta proposta pelo Plano Nacional e também aos valores encontrados para o Brasil e Minas Gerais. Dessa forma, é necessário o desenvolvimento de ações, em todos os níveis de atenção, que favoreçam a adesão ao tratamento da tuberculose e também desenvolver estratégias para acompanhar o tratamento evitando a ocorrência de desfechos desfavoráveis.

Em relação a situação da $\mathrm{TB}$ associada ao HIV percebe-se que o município desenvolveu ações que conseguiram alcançar resultados favoráveis, pois a análise dos testes rápidos de HIV realizados nos casos novos diagnosticados com TB, mostrou que apesar de ainda não atingir $100 \%$ de cobertura, Juiz de Fora apresentou indicadores superiores aos encontrados no Brasil e Minas Gerais e, além disso, superou metas propostas no Plano de Saúde 2014-2017 do município. Outro indicador que se destacou positivamente foi o aumento na porcentagem de cultura de escarro realizada entre os casos de retratamento de TB que foi superior ao observado em nível estadual e nacional. Futuramente, mais estudos serão necessários para acompanhar a evolução desses indicadores contribuindo para o processo de planejamento e desenvolvimento de ações de controle da doença.

\section{REFERÊNCIAS}

ANGELO, J.R. (Re) Produção do espaço urbano de Juiz de Fora - MG e distribuição espacial da tuberculose. 148 f. Dissertação (Mestrado em Ciências na área de Saúde Pública), Escola Nacional de Saúde Pública Sergio Arouca, Fundação Oswaldo Cruz, Rio de Janeiro, 2008.

ARAÚjo, M. JF é a $2^{a}$ em casos de tuberculose em Minas. Tribuna de Minas. 2016. Disponível em https:// tribunademinas.com.br/noticias/cidade/24-03-2016/jf-e-a-2aem-casos-de-tuberculose-em-minas.html. Acesso em 04 de dezembro de 2018.

BARREIRA, D. Os desafios para a eliminação da tuberculose no Brasil. Epidemiologia e Serviços de Saúde, v. 27, n. 1, p. 1-4, Mar. 2018.

BRASIL. Ministério da Saúde. Banco de dados do Sistema Único de Saúde-DATASUS. Disponível em http://www.datasus.gov.br. Acesso em 7 de setembro de 2018.

BRASIL. Ministério da Saúde. Brasil Livre da Tuberculose Plano Nacional pelo Fim da Tuberculose como Problema de Saúde Pública. 2017. Disponível em http://bvsms.saude.gov.br/ bvs/publicacoes/brasil_livre_tuberculose_plano_nacional.pdf Acesso em 6 de setembro de 2018. 
BRASIL. Ministério da Saúde. Implantação do Plano Nacional pelo Fim da Tuberculose como Problema de Saúde Pública no Brasil: primeiros passos rumo ao alcance das metas. 2018. Disponível em http://portalarquivos2.saude.gov.br/images/ pdf/2018/marco/26/2018-009.pdf. Acesso em 20 de agosto de 2018.

BRASIL. Ministério da Saúde. Manual de Recomendações para o controle da Tuberculose no Brasil. 2011. Disponível em http:// bvsms.saude.gov.br/bvs/publicacoes/manual_recomendacoes_ controle_tuberculose_brasil.pdf. Acesso em 17 de janeiro de 2019.

BRASIL. Ministério da Saúde. Panorama da tuberculose no Brasil- Indicadores epidemiológicos e operacionais. 2014. Disponível em http://bvsms.saude.gov.br/bvs/publicacoes/ panorama\%20tuberculose\%20brasil_2014.pdf Acesso em 14 de agosto de 2018 .

BRASIL. Ministério da Saúde. Programa Nacional de Controle da Tuberculose. Disponível em http://bvsms.saude.gov.br/bvs/ publicacoes/ProgramaTB.pdf. Acesso em 07 de setembro de 2018.

BRASIL. Ministério da Saúde. Recomendações para o manejo da coinfeç̧ão TB-HIV em serviços de atenção especializada a pessoas vivendo com HIV/AIDS. 2013. Disponível em: http:// bvsms.saude.gov.br/bvs/publicacoes/recomendacoes_manejo_ coinfeccao_tb_hiv.pdf Acesso em 07 de setembro de 2018.

BRASIL. Mistério da Saúde. Vigilância epidemiológica da tuberculose: Análise de indicadores operacionais e epidemiológicos a partir da base de dados do Sinan versão 5.0. 2016. Disponível em http://portalsinan.saude.gov.br/ images/documentos/Agravos/Tuberculose/Apostila_Curso_ Sinan_2016.pdf. Acesso em 14 de agosto de 2018.

CONSELHO NACIONAL DE SECRETÁRIOS DE SAÚDE. Guia de apoio à gestão estadual do SUS. Disponível em http:// www.conass.org.br/guiainformacao/notas_tecnicas/NT18TUBERCULOSE-Indicadores-epidemiologicos.pdf. Acesso em 04 de dezembro de 2018.

COSTA, R.R., et al. Comparação entre os métodos de OgawaKudoh e Petroff modificado para o cultivo de micobactérias no diagnóstico da tuberculose pulmonar. Einstein, v. 16, n. 2, p. 1-5, Jun. 2018.

INSTITUTO BRASILEIRO GEOGRAFIA ESTATÍSTICA - IBGE. Estimativas da população residente para os municípios e para as unidades da federação. Disponível em https://www.ibge.gov. br/estatisticas-novoportal/sociais/populacao/9103-estimativasde-populacao.html?=\&t=o-que-e. Acesso em: 09 de setembro de 2018.

JUIZ DE FORA. Secretaria de Saúde. Plano de saúde 2014-2017. 2013. Disponível em https://www.pjf.mg.gov.br/conselhos/ cms/arquivos/plano_saude_2014_2017.pdf. Acesso em 04 de dezembro de 2018.
LIMA, M.F.F. O contexto socioambiental da tuberculose pulmonar em Juiz de fora (MG). 275 f. Dissertação (Doutorado em Ciências Ambientais), Instituto de Ciências Biológicas, Universidade Federal de Goiás, Goiás, 2013.

MINAS GERAIS. Secretaria de Estado de Saúde. Plano estadual pelo fim da tuberculose como problema de saúde pública em Minas Gerais 2019-2022. 2019. Disponível em http://www. saude.mg.gov.br/images/noticias_e_eventos/000_2019/jane_ fev_mar/PLANO_ESTADUAL_PELO_FIM_DA_TUBERCULOSE_ COMO_PROBLEMA_DE_SA\%C3\%9ADE_P\%C3\%9ABLICA_EM_ MG.pdf. Acesso em 02 de fevereiro de 2019.

ORGANIZAÇÃO MUNDIAL DA SAÚDE. Global tuberculosis report 2017. Disponível em https://www.who.int/tb/publications/ global_report/gtbr2017_main_text.pdf. Acesso em 04 de dezembro de 2018.

ORGANIZAÇÃO MUNDIAL DA SAÚDE. Global tuberculosis report 2018. Disponível em: http://apps.who.int/iris/bitstream/hand le/10665/274453/9789241565646-eng.pdf?ua=1. Acesso em 04 de dezembro de 2018.

PEREIRA, A., et al. Fatores associados ao óbito e ao abandono do tratamento da tuberculose em um hospital geral do município do Rio de Janeiro, 2007 a 2014. Revista de Epidemiologia e Controle de Infecção, v.8, n. 5, p.1-9, mai. 2018.

PEREIRA, J.C., et al. Perfil e seguimento dos pacientes com tuberculose em município prioritário no Brasil. Revista de Saúde Pública, v.49, n.6, p. 1-12, fev. 2015.

ROCHA, D.S.; ADORNO, R.C.F. Abandono ou Descontinuidade do Tratamento da Tuberculose em Rio Branco, Acre. Saúde e Sociedade, v.21, n. 1, p. 232-245, mar. 2012.

SILVA, F.S., et al. Use of the Ogawa-Kudoh method to isolate mycobacteria in a tuberculosis reference laboratory in northwestern Paraná, Brazil. Brazilian Journal of Pharmaceutical Sciences, v.49, n.3, p. 567-570, jun. 2013.

SULIS,G., et al. Recent developments in the diagnosis and management of tuberculosis. Primary Care Respiratory Medicine, v. 26, n. 16078 , p. 1-8, nov. 2016. 